\title{
Phytopathology"
}

\section{Mycoviruses in Biological Control: From Basic Research to Field Implementation}

\author{
M. D. García-Pedrajas, ${ }^{1, \dagger}$ M. C. Cañizares, ${ }^{1}$ J. L. Sarmiento-Villamil, ${ }^{2}$ A. G. Jacquat, ${ }^{3}$ and J. S. Dambolena ${ }^{3}$
}

${ }^{1}$ Instituto de Hortofruticultura Subtropical y Mediterránea "La Mayora”-Universidad de Málaga-Consejo Superior de Investigaciones Científicas (IHSM-UMA-CSIC), Estación Experimental "La Mayora," 29750 Algarrobo-Costa, Málaga, Spain

${ }^{2}$ Centre d'étude de la Forêt (CEF) and Institut de Biologie Intégrative et des Systèmes (IBIS), Université Laval, Québec, QC G1V 0A6, Canada

${ }^{3}$ Instituto Multidisciplinario de Biología Vegetal (IMBiV-CONICET), Universidad Nacional de Córdoba, Avenida Vélez Sarsfield 1611, Córdoba, X5016GCA, Argentina

Accepted for publication 7 August 2019.

\begin{abstract}
Mycoviruses from plant pathogens can induce hypovirulence (reduced virulence) in their host fungi and have gained considerable attention as potential biocontrol tools. An increasing number of mycoviruses that induce fungal hypovirulence, from a wide variety of taxonomic groups, are currently being reported. Successful application of these viruses in disease management is greatly dependent on their ability to spread in the natural populations of the pathogen. Mycoviruses generally lack extracellular routes of transmission. Hyphal anastomosis is the main route of horizontal mycovirus transmission to other isolates, and conidia of vertical transmission to the progeny. Transmission efficiencies are influenced by both the fungal host and the infecting virus. Interestingly, artificial transfection methods have shown that potential biocontrol mycoviruses often have the ability to infect a variety of fungi. This expands their possible use to the control of pathogens others than those where they were identified. Mycovirus research is also focused on gaining insights into their complex molecular biology and the molecular bases of fungus-virus interactions. This knowledge could be exploited to manipulate the mycovirus and/or the host and generate combinations with enhanced properties in biological control. Finally, when exploring the use of mycoviruses in field conditions, the pathogen life style and the characteristics of the disease and crops affected will deeply impact the specific challenges to overcome, and the development of biocontrol formulations and delivery methods.
\end{abstract}

\section{MYCOVIRUSES EXHIBIT HIGH TAXONOMIC DIVERSITY}

A characteristic feature of mycoviruses is that they generally lack an extracellular phase in their life cycles, depending exclusively on intracellular mechanisms for their transmission. The main routes of mycovirus transmission are through spores (vertical transmission to the progeny) and by hyphal anastomosis (horizontal transmission between different fungal isolates) (Pearson et al. 2009; Xie and Jiang 2014). They are therefore considered intrinsic genetic elements of the fungal isolates that host them and it was thought they had evolved to cause mainly cryptic infections. That notion changed with the identification of Cryphonectria parasitica hypovirus 1 (CHV1) as a mycovirus that had entered the European

${ }^{\dagger}$ Corresponding author: M. D. García-Pedrajas; mariola@eelm.csic.es

*The $\boldsymbol{e}$-Xtra logo stands for "electronic extra" and indicates that one supplementary file is published online.

The author(s) declare no conflict of interest.

(C) 2019 The American Phytopathological Society populations of Cryphonectria parasitica and spread spontaneously, slowing the epidemic of chestnut blight (Heiniger and Rigling 1994). The recognition that mycoviruses can induce hypovirulence (reduced virulence) in their hosts elicited great interest in characterization of viruses from phytopathogenic fungi because of their potential as biocontrol tools. Scores of mycoviruses are now reported. The majority of viruses from filamentous fungi possess either double-stranded RNA (dsRNA) genomes or positive sense (+) singlestranded RNA (ssRNA) genomes with dsRNA replicative intermediates, and can possess a capsid forming true virions or be capsidless. CHV1 is the founding member of the (+) ssRNA mycovirus family Hypoviridae, the first one described whose members lack a capsid (Dawe and Nuss 2013; Hillman and Suzuki 2004). Three other hypoviruses have been identified in $C$. parasitica, named CHV2, CHV3, and CHV4 (Hillman and Suzuki 2004). The isolation of Sclerotinia sclerotiorum hypovirus 1 (SsHV1) and Valsa ceratosperma hypovirus 1 (VcHV1) (Xie et al. 2011; Yaegashi et al. 2012) provided the first evidence that the family Hypoviridae was not restricted to Cryphonectria species as hosts. Two additional families, Narnaviridae and Endornaviridae, comprise capsidless (+) ssRNA mycoviruses. Recently, it has been proposed that unclassified yadokariviruses may conform a new family of capsidless (+) ssRNA mycoviruses, family 
Yadokariviridae (Hisano et al. 2018). Encapsidated (+) ssRNA mycoviruses include families Alphaflexiviridae, Gammaflexiviridae, and Barnaviridae. Mycoviruses with encapsidated dsRNA genomes form the larger group and belong to a wide variety of viral families, including Totiviridae, Partitiviridae, Chrysoviridae, Reoviridae, Megabirnaviridae, and Quadriviridae. In recent years, fungal viruses with (-) ssRNA genomes and with (+) ssRNA genomes possessing genes encoding reverse transcriptase have been described (Ghabrial et al. 2015). A very unusual case is that of Sclerotinia sclerotiorum hypovirulence-associated DNA virus 1 (SsHADV-1), which possesses a circular ssDNA genome ( $\mathrm{Yu}$ et al. 2010). Current metagenomics approaches are rapidly increasing the identification of new mycoviruses and providing compelling evidence of their high abundance and taxonomic complexity.

\section{MYCOVIRUS POTENTIAL ASSOCIATION WITH HYPOVIRULENCE CANNOT BE INFERRED BY TAXONOMIC RELATIONSHIPS}

Major steps in the exploration of mycovirus-based control strategies are illustrated in Figure 1. A well-established hypovirulent effect on the host is the first requisite of a potential biocontrol mycovirus. However, mycoviruses are not infective in the classical sense, lacking extracellular routes of transmission. The question of how to fulfill Koch's postulates, providing definite evidence that a mycovirus is the infectious agent causing host fungus hypovirulence, therefore posed a challenge. The work performed with $\mathrm{CHV} 1$ and $C$. parasitica led the way to solve this problem with the development of infectious cDNA clones for the artificial extracellular infection by prototypical strain CHV1-EP713 (reviewed by Dawe and Nuss 2013). Two different approaches were used. First, a CHV1 full-length cDNA clone was placed under transcriptional regulation of $C$. parasitica sequences to promote synthesis of the virus coding strand upon integration of the infectious clone into the fungal genome. In the second approach, protoplasts of $C$. parasitica were directly transformed with an in vitro-transcribed synthetic copy of the coding RNA strand (Chen et al. 1994). Moleleki et al. (2003) transfected Diaporthe perjunca protoplasts with a synthetic transcript of Diaporthe RNA virus (DaRV1), confirming the feasibility of using this approach with other mycoviruses and hosts. Also using a synthetic transcript, Marzano et al. (2015) successfully transfected Sclerotinia sclerotiorum with a recombinant strain of hypovirus SsHV2. Infectious full-length synthetic clones, in addition to allowing fulfillment of Koch's postulates, has been critical to hypovirus molecular analysis (Dawe and Nuss 2013).

The development of functional infectious clones is nevertheless not a simple task, especially in the case of multisegmented mycoviruses. For encapsidated mycoviruses, inoculation of fungal protoplasts with purified virions, using standard polyethylene glycol-mediated transformation protocols, have been found to work well to initiate infection (Castro et al. 2003; Hillman et al. 2004; Sasaki et al. 2006) and is now an approach of common use. There are two reports of successful infection by transfecting protoplasts directly with purified viral dsRNAs (Jia et al. 2017; Kanhayuwa et al. 2015). Additional work will be required to determine if this very simple method to artificially infect fungal strains could be of more general use.

Natural intracellular transmission mechanisms are also amply used to generate isogenic virus-free and infected fungal strains. For instance, virus-free versions of naturally infected isolates, the socalled "cured" isolates, can be obtained by techniques that rely on selecting progeny or fungal tissue without the virus. The success of these approaches in curing infection appears to be highly variable. As an alternative, mycoviruses can be transmitted to uninfected isolates using the main route of horizontal mycovirus transmission, hyphal anastomosis.
These procedures that exploit natural intracellular transmission pathways, or rely on artificial infection methods, are identifying an increasing number of hypovirulence-associated mycoviruses and show that the taxonomic relationship of a virus cannot be taken as indicative of its effect on the host fungus. Firstly, in addition to the family Hypoviridae, many taxonomic groups with both dsRNA and (+) ssRNA genomes possess members associated with hypovirulence, including families Alphaflexiviridae, Botybirnaviridae, Chrysoviridae, Endornaviridae, Megabirnaviridae, Narnaviridae, Partitiviridae, Reoviridae, and Totiviridae (Ghabrial et al. 2015). Hypovirulence inducing mycoviruses also include Sclerotinia sclerotiorum negative-stranded RNA virus 1 (SsNSRV-1) that possesses a (-) ssRNA genome (Liu et al. 2014), and the geminilike ssDNA virus SsHADV-1 (Yu et al. 2010) (Table 1). Secondly, viruses that are closely related phylogenetically might have very different impacts on the host. There are no mycovirus taxonomic groups that can be considered inextricably linked to hypovirulence, including the family Hypoviridae where species that do not impact virulence have been reported (Hillman and Suzuki 2004; Velasco et al. 2018; Wang et al. 2013). Furthermore, mycovirus effects have been shown to be strain-specific. Thus, different viral strains might behave quite differently and their effects can be further modulated by the particular genetic background of the host fungus strain (Hillman and Suzuki 2004; Hyder et al. 2013; Lin et al. 2007; Vainio and Hantula 2016).

\section{MYCOVIRUS HORIZONTAL AND VERTICAL TRANSMISSION IN THE HOST FUNGUS}

In addition to inducing hypovirulence, mycoviruses requires the ability to infect a large proportion of the pathogen populations in order to be suitable biological control tools. This is strongly dependent on how efficiently they are transmitted to uninfected isolates through hyphal anastomosis, converting them from virulent to hypovirulent, and on how efficiently they are transmitted to the progeny, that defines viral dispersion within the same genetic background. The ability of two isolates to undergo hyphal anastomosis is determined by the complex self/nonself recognition systems or "vegetative incompatibility" (vic) developed by fungal species. Whereas vegetative compatible isolates are able to establish successful hyphal anastomosis, the interaction of vegetative incompatible isolates triggers localized programmed cell death (PCD) which hampers exchange of genetic material (Biella et al. 2002). In the Ascomycota, this process is governed at the molecular level by a series of gene loci collectively known as vic (vegetative incompatibility) or het (heterokaryon incompatibility) (Glass and Dementhon 2006). In C. parasitica, vic loci have been characterized in detail. Transmission studies clearly established that allelic differences at the vic loci negatively affects horizontal mycovirus transmission in this species (Cortesi et al. 2001; Zhang et al. 2014). Disruption analysis of vic genes has confirmed that allelic differences at five of the six vic loci identified in $C$. parasitica contribute to a variable degree to restrict mycovirus transmission (Choi et al. 2012; Zhang et al. 2014).

The transmission rate of mycoviruses in the natural populations of the pathogen to be controlled is expected to depend basically on the host fungus genetics which regulates vegetative incompatibility. This is a concern in the application of mycovirus-based control strategies in plant pathogens with complex vic systems that results in high diversity of vegetative compatibility groups (VCGs) (Jiang et al. 2013; Pearson and Bailey 2013). However, it is becoming increasingly clear that the question of how efficiently a mycovirus can spread to isolates in the natural populations of the fungus is complex. For instance, spread of CHV1 in natural conditions was often found to be more efficient than expected by the complexity of VCGs in field isolates. Specifically addressing this question, 
A

Identification of mycoviruses infecting the pathogen to be controlled

Production of isogenic infected and virus-free strains

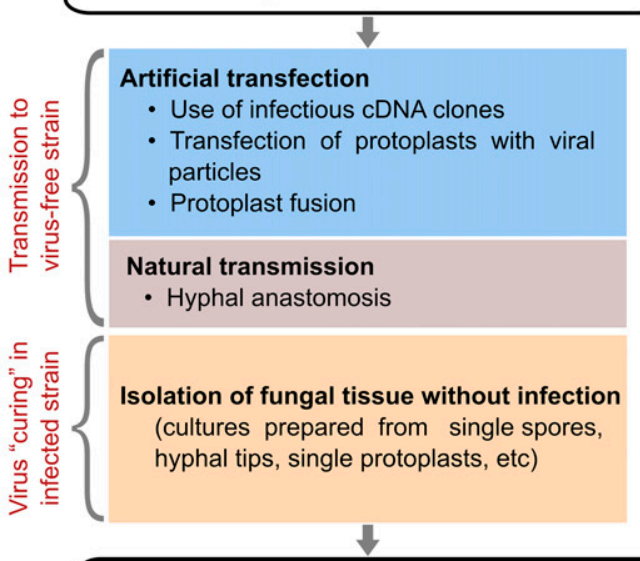

Confirmation of the mycovirus hypovirulent effect in the natural or artificial host fungus
Option 2:

Infection of the pathogen to be controlled with mycoviruses from other fungi using artificial transfection methods

\section{Artificial transfection \\ - Use of infectious cDNA clones \\ - Transfection of protoplasts with viral particles \\ - Protoplast fusion}

B

Analysis of the hypovirulence-inducing mycovirus potential to efficiently spread in the pathogen populations

\section{$\checkmark$}

Mycovirus horizontal transmission to uninfected isolates:

- Main route: hyphal anastomosis

- Generally determined by the complexity of the fungal host vic system

- Mycoviruses can alter host vegetative incompatible reactions favoring horizontal transmission

- Mycoviruses are readily transmitted between incompatible strains in some fungal species
Mycovirus vertical transmission to the progeny:

- Generally transmitted through conidia and frequently excluded from ascospores

- Transmission rates to the progeny vary considerably among mycoviruses

- Mixed infections impact vertical transmission efficiencies of the co-infecting mycoviruses

- The interaction with the antiviral RNAi host machinery influences vertical transmission rates

\section{Molecular manipulation of mycoviruses and/or their hosts to enhance their biological control potential \\ - Disruption of the host fungus vic system to increase mycovirus horizontal transmission \\ - Nuclear integration of a cDNA copy of the mycovirus genome to allow vertical transmission to ascospores and horizontal transmission through sexual crosses \\ - Production of chimeric mycoviruses with improved ecological fitness}

D

Biocontrol formulations:

Development of biocontrol formulations and deployment methods

- The pathogen disease cycle, including main forms of spread and inoculum source, will determine the fungal structures used to carry the biocontrol mycovirus (i.e. spores, hyphae, resistant structures, etc)

- The biocontrol mycovirus should be transferred to local fungal isolates of representative vegetative compatibility groups to prepare the biocontrol formulations to be used
Deployment methods:

- Methods for the successful delivery of biocontrol mycoviruses in the field will vary considerable according the characteristics of the disease and crop(s) affected

- The methodology used for the therapeutic treatment of chestnut cankers with spores of CHV1-infected C. parasitica strains cannot be used as a direct model for other fungal diseases

\section{FIGURE 1}

Schematic representation of major steps in the implementation of control strategies of fungal diseases based on the use of mycoviruses. A, Identification of viruses that reduce the virulence of fungal plant pathogens. Different methods have been developed to produce isogenic infected and virus-free strains to clearly establish the association of the mycovirus under study with hypovirulence. Some of these methods are also used to transfer mycoviruses to other fungal hosts and explore the potential of control strategies based on heterologous mycoviruses. B, Mycoviruses are generally transmitted only by intracellular mechanisms whose characterization is of great importance; efficient horizontal and vertical transmission of the hypovirulence-inducing mycoviruses will determine their ability to spread in the natural populations of the pathogen and therefore their potential as biocontrol agents. C, The increasing availability of tools to molecularly manipulate mycoviruses and their hosts, and the knowledge of the virus-fungus interaction these approaches are generating, is making it possible to genetically manipulate natural mycoviruses and/or their host to produce combinations with improved biological control properties. D, However, even when suitable combinations of mycovirus and fungal pathogen strains for the control of a plant disease become available, their successful deployment in field conditions poses a great challenge. The many problems to overcome, that are yet little addressed in the literature, are related to the life and disease cycle of the pathogen and the characteristics of the disease and the crop(s) affected. 
Brusini and Robin (2013) found that transmission rates were higher on chestnut stems than in the commonly used in vitro assay on synthetic medium. These results suggest that there are environmental factors that favor CHV1 horizontal transmission. In plant pathogenic fungi, other than $C$. parasitica, vic alleles are poorly characterized at the molecular level. Nonetheless, there are rather straightforward approaches to characterize VCG structures in fungal species even in the absence of detail genetic information; pairing of easily obtainable auxotrophic mutants is the most commonly used. Information about VCGs is therefore widely available and it has been exploited to determine the effect of vegetative compatibility/incompatibility on mycovirus transmission in several different host/virus systems. Taken together these studies confirm that, although vegetative incompatibility can be

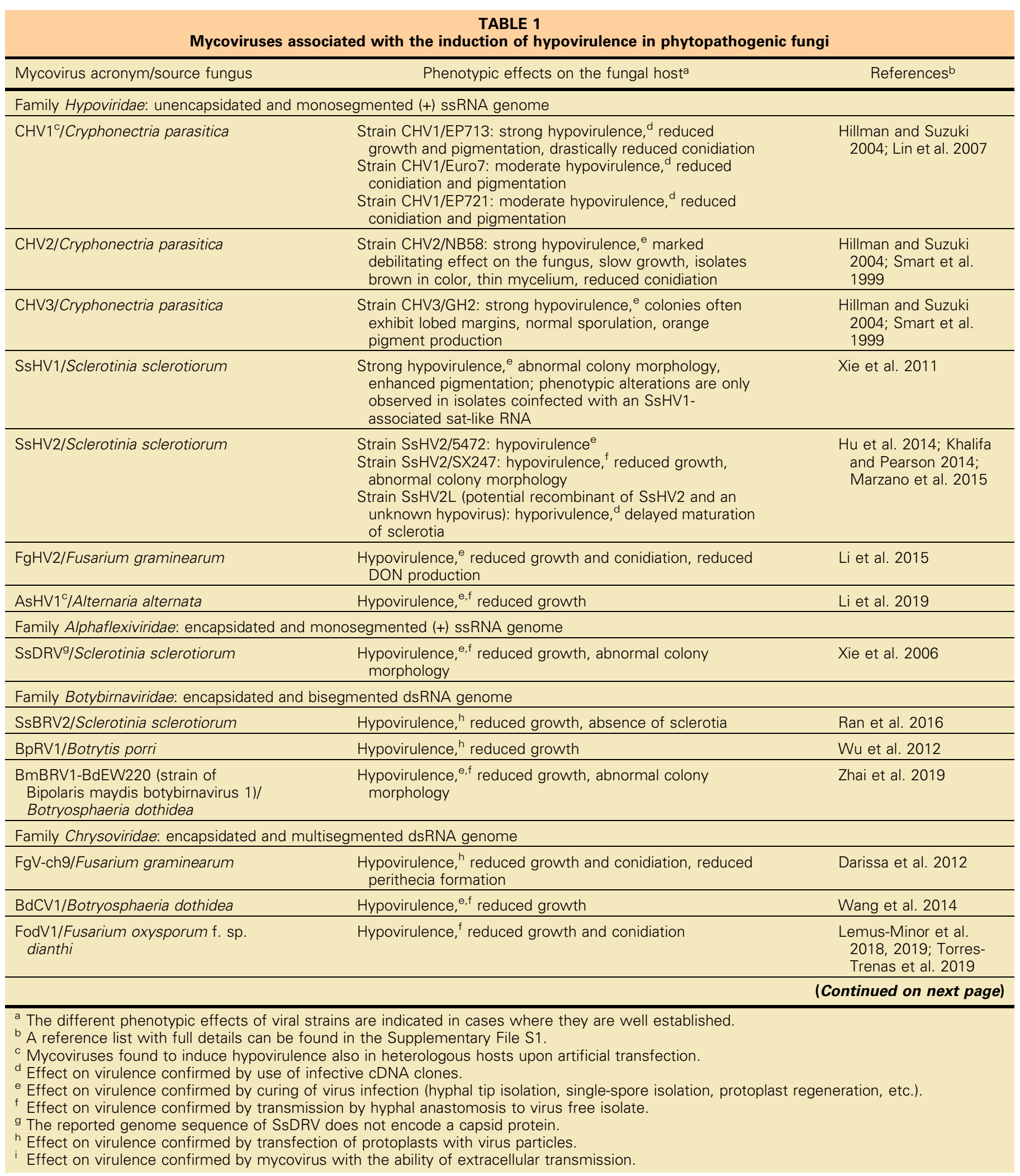


generally regarded as a barrier to mycovirus horizontal transmission, the strength of this barrier can vary considerably. For example, Heterobasidion mycoviruses are readily transmitted even between different Heterobasidion species (Vainio and Hantula 2016; Vainio et al. 2018). Moreover, viruses that seemingly influence their hosts vic reactions have been characterized (Hamid et al. 2018; Wu et al. 2017; Yang et al. 2018). Vegetative incompatibility functions restricting the exchange of genetic material through the induction of localized PCD (Biella et al. 2002). It is plausible therefore that higher mycovirus transmission efficiency in certain fungal species, environmental conditions or with certain mycoviruses might be related to a weakening of PCD responses.

TABLE 1

(Continued from previous page)

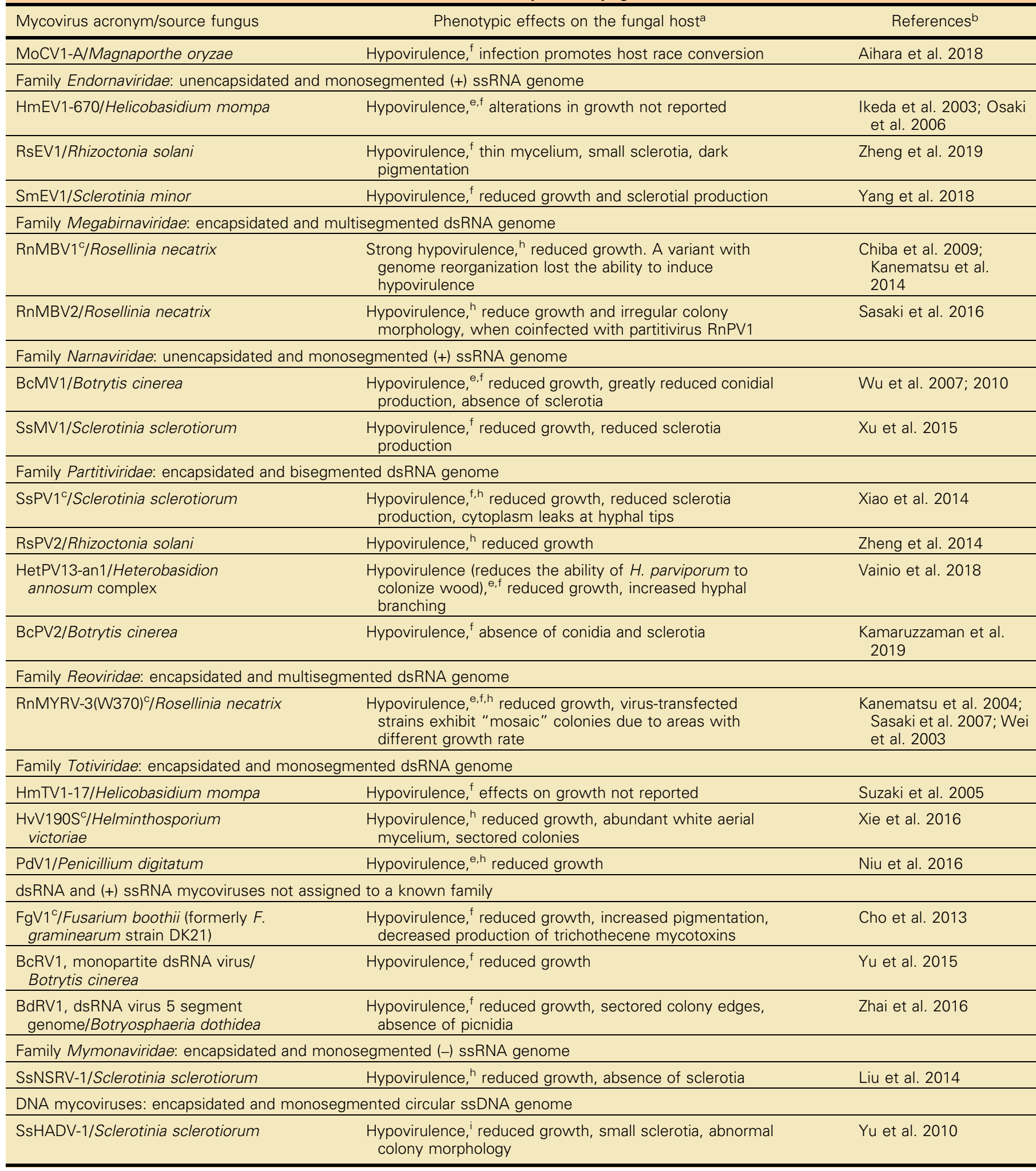


Very interesting in this regard is the work of Wu et al. (2017), which showed that Sclerotinia sclerotiorum mycoreovirus 4 (SsMYR4) infection downregulated critical cellular activities and signaling pathways associated with vegetative incompatibility-mediated PCD, facilitating horizontal transmission of other coinfecting viruses. Mycoviruses with this property can be of great interest for their exploitation in biological control. Analysis of the molecular mechanisms underlying enhanced horizontal transmission will generate knowledge that could be broadly exploited to improve mycovirus-based control strategies.

Mycovirus dispersion is also greatly impacted by transmission rates to the progeny (vertical transmission), which determines the stability of the infection within a genetic background. In C. parasitica, hypoviruses are excluded from ascospores. Analysis of their transmission rates to conidia has provided evidence that poor vertical transmission correlates with limited dispersion of the virus in the host populations. Sparse transmission to the progeny is thought to play an important role in the lack of ecological fitness of CHV2. This hypovirus induces strong hypovirulence in C. parasitica but is transmitted in only 2 to $5 \%$ of the conidia and has a very limited geographical distribution (Hillman and Suzuki 2004). There are systematic studies of mycovirus vertical transmission in other species. Lee et al. (2014) analyzed transmission rates to conidia of four Fusarium graminearum viruses from different viral families. Interestingly, this work revealed that two mycoviruses that induced hypovirulence and growth alterations were more efficiently transmitted than those that caused symptomless infections. Attempts to cure mycovirus infection through the analysis of single-spore cultures have also produced abundant data that confirm that mycovirus transmission rates to conidia can vary considerably among species, and that less impact on the host is not necessarily associated with more efficient vertical transmission. A recent paper by Aulia et al. (2019) has shown that the presence of a coinfecting mycovirus can greatly impact the efficiency of vertical transmission, likely due to suppression of RNA silencing. Exclusion from sexual spores was considered a common feature of mycovirus transmission to the progeny. However, mitoviruses and later on two mycoreoviruses from $C$. parasitica were found to be vertically transmitted through ascospores (Deng et al. 2007b; Polashock et al. 1997). Mycovirus BVX from Botrytis cinerea is also present in ascospores (Pearson and Bailey 2013), and Heterobasidion mycoviruses are transmitted via basidiospores (Vainio and Hantula 2016). It would be of interest to perform more systematic analyses of mycovirus transmission into sexual spores. Sexual reproduction in nature appears to be infrequent but it would be an avenue of mycovirus exchange between different VCGs (Pearson and Bailey 2013). Furthermore, there are fungal species that do not conidiate or in which conidia play a small role in the life cycle. Hence, it would also be of interest to expand current studies of mycovirus vertical transmission to other asexual structures. For example, the sclerotia produced by $S$. sclerotiorum might play an important role in the vertical transmission of mycovirus in this fungal species lacking conidia (Jiang et al. 2013). Recently, transmission of an endornavirus to sclerotia was reported in the related species $S$. minor (Yang et al. 2018).

\section{POTENTIAL CONTROL OF PLANT PATHOGENS WITH MYCOVIRUSES IDENTIFIED IN OTHER FUNGAL SPECIES}

The search of mycoviruses with potential as biological control agents has focused mainly on identifying and characterizing those viral species naturally infecting the fungi to be controlled. However, mycovirus of interest have not been identified in a number of phytopathogenic species with great economic impact and genetic resources available to characterize mycovirus-host interactions.
The use of heterologous mycoviruses could be an alternative in such fungal pathogens. There are a few reports of different fungal species, even phylogenetically distant, naturally infected with strains of the same mycovirus (reviewed by Xie and Jiang 2014). This was an indication that there are viruses with the ability to parasitize a variety of fungi. Natural transmission of mycoviruses between unrelated species is, however, not often encountered. Hence, the artificial transfection methods above described have been exploited to provide clear evidence that mycoviruses can have broad host ranges. For example, the synthetic transcript of CHV1EP713 has been used to artificially infect species closely related phylogenetically to $C$. parasitica, including $C$. radicalis, $C$. havanensis, C. cubensis, and Endothia gyrosa (Chen et al. 1996; van Heerden et al. 2001). In all these fungi, CHV1 was able to replicate and induce hypovirulence and other phenotypic alterations. Furthermore, upon infection, CHV1 was readily transmitted to other isolates of the same species by hyphal anastomosis whereas its effect on sporulation and its vertical transmission to conidia was more variable. Sasaki et al. (2002) used the full-length cDNA clone under fungal regulatory sequences to expand CHV1 infection to other Diaporthales, like Valsa ceratosperma and Phomopsis G-type (teleomorph Diaporthe Nitschke). CHV1 was also able to infect these more distantly related species causing alteration in growth and reduced virulence.

Transfection of protoplasts with viral particles is the method commonly applied to infect new species with encapsidated mycoviruses. Kanematsu et al. (2010) used purified virus particles of Rosellinia necatrix partitivirus 1 (RnPV1) and Rosellinia necatrix mycoreovirus 3 (RnMyR3) to transfect protoplast of Diaporthe sp., C. parasitica, V. ceratosperma, and Glomerella cingulata. Partitivirus RnPV1 was able to establish infection in all four new species, whereas RnMyR3 infected all except $G$. cingulata. Furthermore, RnMyR3 induced attenuated virulence in Diaporthe sp., C. parasitica, and V. ceratosperma, as in its own natural host. Both mycoviruses were readily transmitted by hyphal anastomosis in all new species but vertical transmission to spores was poor. Salaipeth et al. (2014) employed the same approach to transfer Rosellinia necatrix megabirnavirus 1 (RnMBV1) to C. parasitica. Again, RnMBV1 persistently infected $C$. parasitica, inducing similar phenotypic alterations in growth and virulence as in $R$. necatrix. Transfection of protoplasts of $B$. cinerea with purified virions of mycovirus Sclerotinia sclerotiorum partitivirus 1 (SsPV1) also resulted in stable persistent infection and induction of reduced virulence and growth alterations (Xiao et al. 2014). It is worth mentioning here that attempts to infect other species with SsHADV-1 showed that this unusual mycovirus, which can be replicated in and transmitted by mycophagous flies, by contrast, has a rather narrow fungal host range (Yu et al. 2013; Liu et al. 2016).

Protoplast fusion is another approach used to transfer mycoviruses to new hosts. It has the advantage that it does not require any manipulation of the virus and can be used with encapsidated and capsidless species. Lee et al. (2011) successfully transferred mycovirus FgV1-DK21 to $F$. graminearum, $F$. asiaticum, F. oxysporum f. sp. lycopercisi, and $C$. parasitica via protoplast fusion. FgV1-DK21 was able to replicate in all these hosts, even the distantly related $C$. parasitica where it caused reduced virulence and growth alterations that differed from those observed in CHV1 infections.

The works above described, although not numerous, provide evidence that the heterologous virus approach to control plant pathogens could be feasible and worth exploring. First of all, viruses able to replicate in a variety of fungal species appear to be common. Secondly, although mycovirus effects vary with the fungus involved, they tend to retain the ability to induce hypovirulence when transfer to new hosts. Hence, the main factor restricting mycoviruses usually to a single host, or a few closely related hosts, 
could be mainly their dependence on intracellular transmission routes of exchange of genetic material that only take place between individuals of the same species. Furthermore, the ability to reduce fungal virulence is a feature that, although it can be modulated by the host fungus, appears to strongly depend on the viral strain. It should be noted that the inability of SsHADV-1 virions to infect fungal species others than those closely related to its natural host suggests that, when mycovirus spread is not restricted by lack of extracellular transmission routes, other factors may contribute to limit the fungal host range.

\section{THE MYCOVIRUS-FUNGUS INTERACTION: HOST AND VIRAL DEFENSE MECHANISMS}

In the virus-fungus molecular interaction there are two broad aspects to be examined that although linked can be considered separate questions. Firstly, what makes the virus efficiently replicate and persist in the fungal tissues and secondly, which cellular processes and/or signaling pathways are altered in the host resulting in hypovirulence and associated traits. Mycoviral accumulation levels are expected to depend primarily on the host antiviral defenses and how the virus counteracts them. The analysis of these processes might be of particular interest in the case of mycoviruses that, as they lack an extracellular phase in their life cycles, can be considered intrinsic genetic elements of their hosts. RNA silencing (RNAi) is a prominent posttranscriptional gene silencing (PTGS) system that has an ample spectrum of functions in eukaryotes. The key components and general mechanism of RNAi are broadly conserved (Meister and Tuschl 2004; Tomari and Zamore 2005). The process is triggered by the presence of dsRNA that is processed by Dicer or Dicer-like (DCL) proteins into small interfering RNAs (siRNAs). One strand of the siRNA is loaded into an RNA-induced silencing complex (RISC), where the Argonaute or Argonaute-like (AGL) proteins play a central role. The siRNAs guide the RISC to the target RNAs, cleaving them. RNA-dependent RNA polymerases (RDRs) convert single-stranded RNA (ssRNA) into dsRNA thus amplifying the siRNA signals.

In plants and invertebrates RNAi is known to play a critical role in antiviral defense. This PTGS machinery was naturally the first process to focus on when antiviral responses were examined in C. parasitica. This fungal species possesses two Dicer genes and four Argonaute genes. Functional analysis of these genes by production of disruption mutants provided strong evidence that a single Dicer and a single Argonaute were effectively involved in antiviral defense in C. parasitica. Mutants of the Dicer gene $d c l 2$ or the Argonaute gene agl2 challenged with CHV1-EP713 exhibited a severely debilitated phenotype and increased viral RNA levels (Segers et al. 2007; Sun et al. 2009). A later study extended the analysis to additional CHV1 viral strains and highlighted the complexity of RNAi antiviral responses (Zhang et al. 2012). Hypovirus strains CHV1-Euro7 and CHV1-EP721 are highly similar in nucleotide sequence to CHV1-EP713 but cause noticeably milder effects. Additionally, CHV1-EP721 exhibits significantly lower accumulation levels. Zhang et al. (2012) found that both CHV1-Euro7 and CHV1-EP721 showed an increase of the viral accumulation levels in the $d c l 2$ mutant strains but, in contrast with CHV1-EP713, neither of these viral strains significantly induced $d c l 2$ expression. Furthermore, in CHV1-EP721 increased viral levels was not correlated with more debilitating effects on the host. Mycoviruses from other families also exhibit increased viral RNA accumulation and more dramatic phenotypic effects upon deletion of $d c l 2$, including heterologous species artificially transfected to C. parasitica (Chiba et al. 2013a, b; Salaipeth et al. 2014; Segers et al. 2007). C. parasitica RNAi appears not to have a prominent defense role against certain viruses. Very recently, Shahi et al. (2019) showed that RNA levels of Cryphonectria mitovirus
1 (CpMV1) are not affected by deletion of critical components of RNAi machinery, nor does CpMV1 infection upregulate expression of these genes. Members of the genus Mitovirus infect mitochondria and this subcellular localization might allow them to escape RNAi defenses.

The potential antiviral role of RNAi is being examined in an increasing number of fungal pathogens. In Colletotrichum higginsianum, two Dicer genes and two Argonaute genes have been functionally characterized by production of deletion mutants. Phenotypic analysis of wild-type and deletion mutants infected with Colletotrichum higginisanum nonsegmented dsRNA virus 1 showed that, as previously described in $C$. parasitica, a single Dicer and a single Argonaute are involved in the antiviral RNAi response in this species (Campo et al. 2016). Other studies have revealed though that Dicers and Argonautes can have redundant functions in antiviral defense. For example, in S. sclerotiorum the debilitated phenotype observed in double mutants, lacking its two Dicer genes, infected with either a hypovirus or the DNA virus SsHADV1, was reverted by complementation with a single Dicer gene (Mochama et al. 2018). In $F$. graminearum the role of RNAi against three different mycoviruses, FgV1 and FgV2, which induce hypovirulence, and $\mathrm{FgV3}$, that causes latent infections, was investigated (Yu et al. 2018). This species encodes two Dicers and two Argonautes. It was found that a single Argonaute gene, $\mathrm{FgAGO}$ 1, and a single Dicer gene, FgDicer-2, are preferentially upregulated during mycovirus infection but taken together the results of Yu and collaborators point to some functional redundancy of Dicers and Argonautes in F. graminearum and virus-specific responses (Yu et al. 2018). Functional analysis of the three Argonautes of Pyricularia oryzae (Magnaporthe oryzae) has led to the intriguing result that one of them interferes with RNAi (Nguyen et al. 2018). These authors found that deletion of MoAGO2 resulted in a dramatic decrease in the accumulation levels of two mycoviral RNAs. Furthermore, this decrease in viral RNA levels did not occurred in a double mutant lacking both MoAGO2 and the Dicer MoDCL2, confirming that the strong silencing of mycoviruses in the $\Delta$ moago 2 strain is RNAi-dependent. In Verticillium dahliae, we have found that viral RNA levels of a partitivirus (Cañizares et al. 2014) and an unclassified (+) ssRNA mycovirus (Cañizares et al. 2018) are not affected by deletion of Dicer or Argonaute genes. This species possesses two Dicer genes and two Argonaute genes and even in double mutants fully lacking Dicer or Argonaute activity, accumulation levels of both mycoviruses were very similar to those exhibited by the wild-type strain (M. C. Cañizares and M. D. García-Pedrajas, unpublished data).

Functional characterization of RNAi components through analysis of virus-infected disruption mutants is providing evidence that antiviral RNAi responses might be more significant in some fungal species and/or against certain mycoviruses. However, it is a rather labor-intensive approach. Presence of virus-derived siRNAs (vsiRNAs) is considered the hallmark of an active antiviral RNAi mechanism. Detection of vsiRNAs can therefore provide indirect evidence of the antiviral role of RNAi in mycovirus-fungus interactions. Nowadays, next generation sequencing is increasingly used to characterize vsiRNAs populations as a simple way to show that mycoviral infection triggers an antiviral RNAi response in the corresponding fungal species. Interestingly, some of these studies have shown that vsiRNAs could target fungal host-specific genes thus inducing their silencing (Ozkan et al. 2017; Wang et al. 2016a). RNAi antiviral responses could therefore modulate fungal cellular processes through this molecular mechanism contributing to the phenotypic effects of mycovirus infection.

Viruses have evolved strategies to counteract the host RNAi responses including encoding RNA silencing suppressors (RSSs). Regarding how mycoviruses defend themselves from antiviral fungal defenses, little information is yet available in mycovirus-fungus interactions. There is one exception to this, the RSS 
protein p29 encoded by CHV1-EP713 which has been studied in detail. The ability of the p29 suppressor protein to enhance viral RNA accumulation of homologous and heterologous viruses has been confirmed by multiple approaches, including coinfection analyses and p29 transgenic expression studies (Chiba et al. 2013b; Segers et al. 2006; Sun et al. 2006; Suzuki et al. 2003). The mechanism of action of p29 likely involves downregulation of the RNAi antiviral response that involves increased expression of $d c l 2$ and agl2. Mycovirus RnMyRV3 from Rosellinia necatrix also encodes an RSS, termed VP10, but its mode of action is unclear (Yaegashi et al. 2013b). Interestingly, large-scale sequencing of vsiRNAs and bioinformatic analyses showed that the RNAi genes Dicer1 and RDR5 of $F$. graminearum could be among the potential targets of hypoviruses FgHV1 and FgHV2 derived siRNAs (Wang et al. 2016a). This result suggests that degradation of RNAi gene transcripts targeted by hypovirus siRNAs could be a strategy to suppress RNAi antiviral responses in addition to the role played by RSS proteins.

\section{THE MYCOVIRUS-FUNGUS INTERACTION: HOST CELLULAR PROCESSES ALTERED BY VIRUS INFECTION}

Comparison of the transcription patterns of isogenic virus-free and virus-infected fungal strains is an approach used in an increasing number of studies to provide insights into basic aspects of the virus-fungus interaction. Mycoviruses that induce hypovirulence often cause diverse growth alterations. The pleiomorphic nature of these alterations suggested that hypovirulence-inducing mycoviruses interfere with host signaling pathways. Using microarray technology, Dawe et al. (2004) showed that around half of the $C$. parasitica genes whose expression was altered by CHV1 infection, exhibited similar changes in expression in deletion mutants lacking G-protein $\alpha$-subunit or $\beta$-subunit genes. CHV 1 also alters the expression of transcription factor (TF) genes, very interestingly, it downregulates the expression of cpst 12 encoding a homolog of the Saccharomyces cerevisiae TF Ste12, a key target of MAPKs signaling and critical for invasive growth and mating. Moreover, the differential gene expression pattern induced by CHV1 infection is very similar to that induced by cpst 12 deletion (Deng et al. 2007a). These results uncovered a probable coordination of G-protein and MAPK signaling pathways in the regulation of TFs involved in virulence in $C$. parasitica. Nuskern et al. (2018) confirmed that CHV1 infection induces epigenetic changes. These authors analyzed the effect of six CHV1 strains on the global cytosine methylation level of three different $C$. parasitica isolates finding that hypovirus infection induces DNA hypermethylation in $C$. parasitica, and that higher methylation levels correlate with more severe phenotypic effects. Other $C$. parasitica cellular processes have been identified as altered in the presence of CHV1. For example, transcriptomic as well as proteomic approaches showed that CHV1 infection affects host secretory pathways and vesicle-mediated transport systems, and upregulates stress responses (Allen et al. 2003; Kazmierczak et al. 2012; Wang et al. 2016b). In summary, hypovirulence and associated traits caused by CHV1 infection of $C$. parasitica is likely a consequence of virus interference with multiple secretory and regulatory mechanisms.

$F$. graminearum is another plant pathogen in which global responses to mycovirus infection have been characterized in some detail. In the first of these studies, Kwon et al. (2009) used a proteomic approach to characterize changes in protein host profiles in response to mycovirus FgV1-DK21, which induces hypovirulence and reduced mycotoxin production. This work showed upregulation of proteins involved in differentiation processes and downregulation of proteins with antioxidant properties and involved in glycolysis in the infected strain. Cho et al.
(2012) analyzed $F$. graminearum transcriptional responses to the same hypovirulence-associated mycovirus with similar results to the above described responses of $C$. parasitica to CHV1. Thus, FgV1 caused upregulation of genes associated with cAMPmediated signaling, the transcriptional and translational machineries as well as ABC transporters. Genes involved in transport systems, and primary and secondary metabolism were, on the other hand, downregulated by FgV1 infection. Later on, using RNA-seq technology, Lee et al. (2014) compared the transcriptomic response of $F$. graminearum to FgV1-DK21 and three additional viruses, $\mathrm{FgV} 2, \mathrm{FgV} 3$, and FgV4. These mycoviruses are from different families and differed too in their host effects. Similarly to FgV1DK21, FgV2 induces reduced virulence and growth changes, whereas FgV3 and FgV4 cause symptomless infections. Unexpectedly, Lee et al. (2014) found that the mycoviruses that had higher impact on host transcription profiles were $\mathrm{FgV} 1$ and $\mathrm{FgV} 4$. $\mathrm{FgV} 2$, although inducing very noticeable phenotypic changes in $F$. graminearum, had clearly less impact on host gene expression. Hence, a greater impact on the host phenotype does not necessary correlate with a bigger impact on its transcription patterns. Remarkably, each mycovirus affected the expression of a different set of fungal genes. For example, in all four interactions the expression of TF genes was significantly changed but each mycovirus regulated a different set of TFs (Lee et al. 2014). The transcriptional response of most key components of the RNAi machinery was also virus-specific.

Wang et al. (2018) recently evaluated the transcriptional response of Botryosphaeria dothidea, the causal agent of pear ring rot, to a chrysovirus and a partitivirus potentially associated with hypovirulence and growth alterations. Similar to the above described transcriptional responses of $C$. parasitica to $\mathrm{CHV}-1$ and $F$. graminearum to $\mathrm{FgV1}$, infection of $B$. dothidea with either mycovirus led to upregulation of genes involved in transcription and signal transduction pathways such as MAPK signaling and stress responses. The expression of key genes of the RNAi machinery was also induced in the infected $B$. dothidea strain. In another recent study, Vainio et al. (2018) reported the effect of Heterobasidion partitivirus 13 (HetPV13-an1) infection on the phenotype and gene expression patterns of $H$. parviporum, a conifer pathogen. It was found that although HetPV13-an1 causes hypovirulence and associated phenotypic alterations, it affects host gene expression patterns in a very different manner than other hypovirulenceinducing mycoviruses. HetPV13-an1 appeared to greatly impact host metabolism; the infected strain showed altered carbohydrate and amino acid metabolism and upregulation of alternative metabolic pathways. Furthermore, HetPV13-an1 infection repressed several potential components of the RNAi machinery (Vainio et al. 2018). The DNA virus SsHADV1 has the very unusual particularity within the mycoviruses that it can infect intact fungal cells as viral particles. The transcriptional response of its host, S. sclerotiorum, to early stages of SsHADV1 infection has also been characterized (Ding et al. 2019). Analysis of the functional categories of genes differentially expressed in pre- and postinfection hyphae has led Ding et al. (2019) to propose that SsHADV1 breaks through the fungal cell wall and use the host Rassmall $\mathrm{G}$ protein signal transduction pathway to modulate changes in metabolism that provides the energy for its own invasion and proliferation.

Current knowledge of fungal transcriptional responses to viral infection allows concluding that diverse molecular mycovirus-host interactions might have a few common features. For instance, mycovirus infections, including symptomless infections, generally result in extensive host transcriptional reprogramming. Alteration of the expression of genes encoding transcription factors and components of signal transduction pathways appears to be another fairly common response to mycovirus infection. However, it is also evident that the responses observed are, to a large extent, 
mycovirus-host-specific. The regulation of fungal virulence and development is a very complex process that involves the interplay of multiple signaling pathways, regulatory mechanisms and cellular processes. Hence, different mycoviruses can interfere with different sets of genes with similar phenotypic consequences for the host.

\section{ENGINEERING MYCOVIRUSES AND/OR THEIR FUNGAL HOSTS TO ENHANCE THE POTENTIAL OF BIOLOGICAL CONTROL STRATEGIES}

Our understanding of the molecular biology of mycoviruses and their hosts is rapidly increasing. In this scenario, manipulating the virus and/or the fungus with the aim of producing the best combinations for effective disease control is becoming possible. For instance, molecular approaches have been used in the extensively studied $C$. parasitica-CHV1 interaction in an attempt to increase the potential of mycovirus dispersion (recently reviewed by Rigling and Prospero 2018). The nuclear integration of a full-length cDNA copy of CHV1 provides a strategy to enhance vertical and horizontal transmission. It results in transmission of the virus to ascospores, and this in turn allows horizontal transmission through sexual crosses regardless of vegetative incompatibility. Field trials have nonetheless failed to support the better performance of $C$. parasitica strains harboring a nuclear copy of CHV1 in spreading the virus (Root et al. 2005). In a different approach, "super donor" C. parasitica strains able to transfer CHV1 to isolates from a variety of VCGs have been produced by disrupting vic loci known to restrict mycovirus transmission. These strains were expected to enhance the effective delivery of hypoviruses in $C$. parasitica populations (Zhang and Nuss 2016). A recent study provided evidence that biocontrol formulations based on "super donor" strains worked more efficiently in the transmission of hypovirus in an American chestnut forest setting with highly divergent C. parasitica populations (Stauder et al. 2019).

The knowledge gained through the functional analysis of virus sequences could be used, on the other hand, to produce chimeric mycoviruses with enhanced properties as biocontrol tools. For example, the potential lack of ecological fitness of the fungal strains harboring biocontrol viruses poses a major concern. Viruses that induce hypovirulence usually have a negative impact on vegetative growth. This might affect their ecological fitness and therefore the spread and prevalence of the biological control agent in natural conditions. The association of hypovirulence with alterations in vegetative growth is common to the extent that these morphological changes are referred to as hypovirulence-associated traits. It should be taken into account though that many mycoviruses that induce hypovirulence were first selected for characterization because their host strains exhibited obvious growth alterations. This might bias the screening toward mycoviruses that affect both vegetative growth and virulence, whereas viruses that only affect pathogenic development would escape notice. Furthermore, mycoviruses that induce growth changes allow virus-free from infected isolates to easily be distinguished, thus providing a simpler experimental system. Some mycoviruses could however affect signaling pathways and cellular processes exclusively associated with pathogenic development. Functional analysis of the prototypical CHV1-EP13 strain sequences has allowed mapping genome regions associated with specific effects on the host (reviewed by Dawe and Nuss 2013). These analyses reveal that it might be possible to fine tune the effect on the host through the molecular manipulation of the mycovirus, uncoupling hypovirulence from alterations in growth. Less impact on the host growth could indeed be an advantage for the dispersion of the mycovirus used as biological control agent in natural conditions (Robin et al. 2010). It is true that the debilitating effect on the host growth might be a factor contributing to disease control by reducing the ability of the virus-infected fungus to colonize plant tissue (Vainio et al. 2018). However, this is not necessary a general scenario and in many pathogens molecular mechanisms critical for pathogenic development but with no impact on fungal growth have been elucidated. The key question in the molecular manipulation of a mycovirus would be to achieve the proper balance between the factors that makes the mycovirus successful in preventing disease, and those that contribute to its ecological fitness allowing it to become prevalent in the pathogen populations. To produce transgenic viruses with enhanced properties as biocontrol tools is doubtless a difficult task. It requires deep understanding of the complex mycovirus molecular biology and extensive field trials. However, the potential of this approach should not be disregarded.

\section{THE BROAD IMPLEMENTATION OF MYCOVIRUS- BASED CONTROL STRATEGIES FACES MULTIPLE CHALLENGES}

The confirmation that spread of CHV1 was lowering the incidence of chestnut blight in European stands led to the implementation of a program of artificial introduction of CHV1infected strains to control $C$. parasitica, which remains active nowadays in Europe. The disease chestnut blight has features that favor mycovirus-based control strategies. C. parasitica affects a tree species causing necrotic lesions in the bark (cankers), which on an individual tree are usually formed from a single point of infections. Treatment of these cankers with spores of hypovirulent strains harboring the hypovirus can halt their development (Hillman and Suzuki 2004; Rigling and Prospero 2018). In biological control strategies, the ideal situation is that, once introduced, the biocontrol agent spreads independently and persists in the ecosystem exerting continuous control. Chestnut blight control offers the possibility of a therapeutic hypovirus treatment of easily identifiable localized symptoms. To improve hypovirus formulations, vic types in the area to be treated are determined and the hypovirus is transmitted to local fungal isolates by hyphal anastomosis using an in vitro transmission assay. The inoculum thus prepared is used to fill holes made at the margins of the cankers with a cork borer. The therapeutic use of CHV1-infected $C$. parasitica strains works well to lower the incidence of chestnut blight in Europe but mostly failed in North America. The higher VCG diversity of the $C$. parasitica North American populations might contribute to these differences (Dawe and Nuss 2013; Milgroom and Cortesi, 2004). However, it is becoming increasingly clear that the efficient spread of mycoviruses upon introduction through hypovirulent infected stains might depend on multiple factors.

There are currently a number of fungal pathogens in which mycoviruses with good potential as biocontrol tools are available. The vegetative incompatibility system of the fungal pathogen to be controlled is frequently the first concern in the potential field application of mycoviruses as a complex VCG structure might hamper spread of the mycovirus to natural fungal isolates. How to favor mycovirus horizontal transmission is therefore an active area of research (Xie and Jiang 2014). There are, however, other key factors that will determine the approach used to deliver hypovirulence-inducing mycoviruses in the field and their ability to spread upon introduction and effectively control the disease. For instance questions related to the life and disease cycle of the pathogen and the characteristics of the disease and crop(s) affected. It could be mentioned in the first place that the simplest way to implement the use of mycoviruses is probably as a therapeutic treatment of visible symptoms but this is an option only in a limited number of plant diseases. Also, in contrast with woody plant diseases, herbaceous crops that are harvested after each growing season might not offer favorable conditions to the colonization of pathogen populations by the virus in field conditions (Jiang et al. 2013). Necrotrophic and biotrophic fungal pathogens can also offer 
very different contexts for the mycovirus' ability to disperse in the local fungal populations in time to effectively control the disease. The mycovirus-fungus formulations and the field delivery methods might vary considerably in soilborne and airborne pathogens and depending on the main agents of dispersal of the disease and inoculum source. Pathogens that survive as resistant structures in the absence of the host plant likely have less chance of the hypovirulence transmission. In summary, the management of C. parasitica with CHV1-infected hypovirulent strains does not necessarily provide a general model to devise formulations of the biological control agent and delivery methods, or to predict potential challenges to the implementation of this type of control strategies. A few examples of different scenarios are considered below to illustrate how the life cycle of the pathogen and the characteristics of the crop(s) affected can impact the practical application of mycovirus-based control strategies, a question that has been so far rarely addressed in the literature.

In the genus Heterobasidion, which includes very devastating conifer pathogens, horizontal virus transmission takes place readily between different species (Vainio and Hantula 2016). In this regard, Heterobasidion species are well suited for the use of biocontrol mycoviruses. Recently, a promising Heterobasidion partitivirus, HetPV3-an1, with a strong debilitating effect on the host was reported (Vainio et al. 2018). H. parviporum can spread from tree to tree via root contacts in the form of vegetative mycelium, whereas the basidiospores gain entry through fresh stump surfaces and stem wounds. In their analysis of HetPV3-an1, Vainio et al. (2018) confirmed that it reduces the ability of $H$. parviporum to grow in spruce trees by colonizing wood plugs with mycelium of the hypovirulent strain and inserting them into holes drilled in the root collar of large spruce trees. In a previous study, Vainio et al. (2013) introduced a virus-infected $H$. parviporum strain by spraying freshly exposed stumps exhibiting visible wood decay with liquid mycelial preparations. Interestingly, after three growing seasons the artificially introduced $H$. parviporum strain could not be detected but the mycovirus it carried had been transmitted to indigenous strains. These analyses provide clues about how mycoviruses could be deployed for Heterobasidion disease control.

$R$. necatrix is a soilborne pathogen that causes white root rot in a variety of crops including fruit trees and grapevine and in which mycoviruses of interest in biological control are available (Chiba et al. 2009; Kanematsu et al. 2004). The main source of $R$. necatrix infection is the mycelium, with the disease transmitted to neighboring trees by root contact (Pliego et al. 2012). Usually, affected orchards exhibit specific field locations where symptoms are repeatedly observed. These disease patches could provide a workable area in which to attempt mycovirus-based control strategies. Yaegashi et al. (2013a) devised a way to inoculate apple trees with infected and virus-free incompatible $R$. necatrix strains to analyze mycovirus epidemiology. For that, the fungus was cultured on apple twigs that were then tied to apple roots. Interestingly, Yaegashi et al. (2013a) found that 3 years after introducing the mycovirus in the orchard, it had been transmitted between the two incompatible strains used in the study and, what is more, these strains had become infected with other mycoviruses of unknown origin. This is an indication that horizontal virus transmission can occurs between incompatible isolates of $R$. necatrix in nature.

$S$. sclerotiorum is another plant pathogen which has become a model system for characterization of fungus-mycovirus associations. Mycoviruses of interest have been described in S. sclerotiorum. Furthermore, some of them are known to be transmitted between vegetative incompatible isolates (Hamid et al. 2018; Wu et al. 2017). Yet, a major issue with the use of mycoviruses to control this pathogen is that most of its hosts are herbaceous crops that are harvested after each growth season (Jiang et al. 2013). A particular example within $S$. sclerotiorum mycoviruses is represented by
SsHADV-1, which is infectious as viral particles. Yu et al. (2013) found that spraying rapeseed plants with either virus particles or infected mycelium functioned well both as a preventive and therapeutic treatment against $S$. sclerotiorum. It is unclear whether a mycovirus with these characteristics could be used as a typical biological control agent that has the ability to persist in the ecosystem upon introduction. Nevertheless, its use as a biofungicide that could be sprayed periodically as part of a disease control program might be an interesting step in the exploitation of mycovirus to control $S$. sclerotiorum. The case of SsHADV-1 is very specific though, whereas the vast majority of mycoviruses can only be delivered in the plant pathogen natural populations through infected fungal strains.

In $F$. graminearum, the main causal agent of Fusarium head blight and a concern for its production of mycotoxins, several hypovirulence inducing mycoviruses are well characterized (Cho et al. 2012; Li et al. 2015). However, there are no thoughts yet in the literature regarding how to implement their use to control a disease that affects annual grain crops, mostly wheat and barley, and can be devastating in a short period of time. Similar challenges might pose the use of mycoviruses to control Magnaporthe oryzae, the most devastating pathogen of rice.

Current knowledge indicates that mycoviruses could be applied to the control of a wide variety of fungal pathogens. This includes fungi with or without natural viral infections of interest, as it has been shown that mycoviruses frequently have broad host ranges and retain the ability to induce hypovirulence when artificially transferred to heterologous hosts. Information regarding the role of vic genes in restricting horizontal transmission, and the characterization of mycovirus that lower vegetative incompatibility barriers, can be exploited to improve the ability of biocontrol viruses to efficiently spread in the natural populations of the pathogen. This might be of particular importance to expand their application to the control of fungal pathogens with complex vic systems. Exploring the use of mixed infections, for example to combine a virus that favor the horizontal and/or vertical transmission with the mycovirus inducing the desired effect on virulence, is also emerging as an interesting topic to explore. In summary, there have been great advances in the exploration of mycoviruses as potential biological agents. Currently, diverse mycoviruses with good potential to control a variety of pathogens are available, and ways to manipulate virus-fungus interactions to improve their properties are being devised. In our view, the real challenge will be to develop field implementation methods that take into account aspects such as main inoculum source and routes of infection, plant colonization modes and disease symptoms and, very importantly, the characteristic of the crops affected.

\section{LITERATURE CITED}

Allen, T. D., Dawe, A. L., and Nuss, D. L. 2003. Use of cDNA microarrays to monitor transcriptional responses of the chestnut blight fungus Cryphonectria parasitica to infection by virulence-attenuating hypoviruses. Eukaryot. Cell 2:1253-1265.

Aulia, A., Andika, I. B., Kondo, H., Hillman, B. I., and Suzuki, N. 2019. A symptomless hypovirus, CHV4, facilitates stable infection of the chestnut blight fungus by a coinfecting reovirus likely through suppression of antiviral RNA silencing. Virology 533:99-107.

Biella, S., Smith, M. L., Aist, J. R., Cortesi, P., and Milgroom, M. G. 2002. Programmed cell death correlates with virus transmission in a filamentous fungus. Proc. R. Soc. B 269:2269-2276.

Brusini, J., and Robin, C. 2013. Mycovirus transmission revisited by in situ pairings of vegetatively incompatible isolates of Cryphonectria parasitica. J. Virol. Methods 187:435-442.

Campo, S., Gilbert, K. B., and Carrington, J. C. 2016. Small RNA-based antiviral defense in the phytopathogenic fungus Colletotrichum higginsianum. PLoS Pathog. 12:e1005640.

Cañizares, M. C., López-Escudero, F. J., Pérez-Artés, E., and García-Pedrajas, M. D. 2018. Characterization of a novel single-stranded RNA mycovirus related to invertebrate viruses from the plant pathogen Verticillium dahliae. Arch. Virol. 163:771-776. 
Cañizares, M. C., Peréz-Artés, E., and García-Pedrajas, M. D. 2014. The complete nucleotide sequence of a novel partitivirus isolated from the plant pathogenic fungus Verticillium albo-atrum. Arch. Virol. 159:3141-3144.

Castro, M., Kramer, K., Valdivia, L., Ortiz, S., and Castillo, A. A. 2003. Double-stranded RNA mycovirus confers hypovirulence-associated traits to Botrytis cinerea. FEMS Microbiol. Lett. 228:87-91.

Chen, B., Chen, C. H., Bowman, B. H., and Nuss, D. L. 1996. Phenotypic changes associated with wild-type and mutant hypovirus RNA transfection of plant pathogenic fungi phylogenetically related to Cryphonectria parasitica. Phytopathology 86:301-310.

Chen, B., Choi, G. H., and Nuss, D. L. 1994. Attenuation of fungal virulence by synthetic infectious hypovirus transcripts. Science 264(5166):17621764.

Chiba, S., Lin, Y. H., Kondo, H., Kanematsu, S., and Suzuki, N. 2013a. Effects of defective interfering RNA on symptom induction by, and replication of, a novel partitivirus from a phytopathogenic fungus, Rosellinia necatrix. J. Virol. 87:2330-2341.

Chiba, S., Lin, Y. H., Kondo, H., Kanematsu, S., and Suzuki, N. 2013b. A novel victorivirus from a phytopathogenic fungus, Rosellinia necatrix, is infectious as particles and targeted by RNA silencing. J. Virol. 87: 6727-6738.

Chiba, S., Salaipeth, L., Lin, Y. H., Sasaki, A., Kanematsu, S., and Suzuki, N. 2009. A novel bipartite double-stranded RNA mycovirus from the white root rot fungus Rosellinia necatrix: Molecular and biological characterization, taxonomic considerations, and potential for biological control. J. Virol. 83:12801-12812.

Cho, W. K., Yu, J., Lee, K. M., Son, M., Min, K., Lee, Y. W., and Kim, K. H. 2012. Genome-wide expression profiling shows transcriptional reprogramming in Fusarium graminearum by Fusarium graminearum virus 1DK21 infection. BMC Genomics 13:173.

Choi, G. H., Dawe, A. L., Churbanov, A., Smith, M. L., Milgroom, M. G., and Nuss, D. L. 2012. Molecular characterization of vegetative incompatibility genes that restrict hypovirus transmission in the chestnut blight fungus Cryphonectria parasitica. Genetics 190:113-127.

Cortesi, P., McCulloch, C. E., Song, H. Y., Lin, H. Q., and Milgroom, M. G. 2001. Genetic control of horizontal virus transmission in the chestnut blight fungus, Cryphonectria parasitica. Genetics 159:107-118.

Dawe, A. L., and Nuss, D. L. 2013. Hypovirus molecular biology: From Koch's postulates to host self-recognition genes that restrict virus transmission. Adv. Virus Res. 86:109-147.

Dawe, A. L., Segers, G. C., Allen, T. D., McMains, V. C., and Nuss, D. L. 2004. Microarray analysis of Cryphonectria parasitica $\mathrm{G} \alpha$ - and $\mathrm{G} \beta \gamma$ signalling pathways reveals extensive modulation by hypovirus infection. Microbiology 150:4033-4043.

Deng, F., Allen, T. D., Hillman, B. I., and Nuss, D. L. 2007b. Comparative analysis of alterations in host phenotype and transcript accumulation following hypovirus and mycoreovirus infections of the chestnut blight fungus Cryphonectria parasitica. Eukaryot. Cell 6:1286-1298.

Deng, F., Allen, T. D., and Nuss, D. L. 2007a. Ste12 transcription factor homologue CpST12 is down-regulated by hypovirus infection and required for virulence and female fertility of the chestnut blight fungus Cryphonectria parasitica. Eukaryot. Cell 6:235-244.

Ding, F., Cheng, J., Fu, Y., Chen, T., Li, B., Jiang, D., and Xie, J. 2019. Early transcriptional response to DNA virus infection in Sclerotinia sclerotiorum. Viruses 11:278.

Ghabrial, S. A., Castón, J. R., Jiang, D., Nibert, M. L., and Suzuki, N. 2015. 50-plus years of fungal viruses. Virology 479:356-368.

Glass, N. L., and Dementhon, K. 2006. Non-self recognition and programmed cell death in filamentous fungi. Curr. Opin. Microbiol. 9:553-558.

Hamid, M. R., Xie, J., Wu, S., Maria, S. K., Zheng, D., Assane Hamidou, A., Wang, Q., Cheng, J., Fu, Y., and Jiang, D. 2018. A Novel deltaflexivirus that infects the plant fungal pathogen, Sclerotinia sclerotiorum, can be transmitted among host vegetative incompatible strains. Viruses 10:e295.

Heiniger, U., and Rigling, D. 1994. Biological control of chestnut blight in Europe. Annu. Rev. Phytopathol. 32:581-599.

Hillman, B. I., Supyani, S., Kondo, H., and Suzuki, N. 2004. A reovirus of the fungus Cryphonectria parasitica that is infectious as particles and related to the coltivirus genus of animal pathogens. J. Virol. 78:892-898.

Hillman, B. I., and Suzuki, N. 2004. Viruses of the chestnut blight fungus, Cryphonectria parasitica. Adv. Virus Res. 63:423-473.

Hisano, S., Zhang, R., Faruk, M. I., Kondo, H., and Suzuki, N. 2018. A neovirus lifestyle exhibited by a (+)ssRNA virus hosted in an unrelated dsRNA virus: Taxonomic and evolutionary considerations. Virus Res. 244:75-83.

Hyder, R., Pennanen, T., Hamberg, L., Vainio, E. J., Piri, T., and Hantula, J. 2013. Two viruses of Heterobasidion confer beneficial, cryptic or detrimental effects to their hosts in different situations. Fungal Ecol. 6:387-396.

Jia, H., Dong, K., Zhou, L., Wang, G., Hong, N., Jiang, D., and Xu, W. 2017. A dsRNA virus with filamentous viral particles. Nat. Commun. 8:168.
Jiang, D., Fu, Y., Li, G., and Ghabrial, S. A. 2013. Viruses of the plant pathogenic fungus Sclerotinia sclerotiorum. Adv. Virus Res. 86:215-248.

Kanematsu, S., Arakawa, M., Oikawa, Y., Onoue, M., Osaki, H., Nakamura, H., Ikeda, K., Kuga-Uetake, Y., Nitta, H., Sasaki, A., Suzaki, K., Yoshida, K., and Matsumoto, N. 2004. A reovirus causes hypovirulence of Rosellinia necatrix. Phytopathology 94:561-568.

Kanematsu, S., Sasaki, A., Onoue, M., Oikawa, Y., and Ito, T. 2010. Extending the fungal host range of a partitivirus and a mycoreovirus from Rosellinia necatrix by inoculation of protoplasts with virus particles. Phytopathology 100:922-930.

Kanhayuwa, L., Kotta-Loizou, I., Ozkan, S., Gunning, A. P., and Coutts, R. H. 2015. A novel mycovirus from Aspergillus fumigatus contains four unique dsRNAs as its genome and is infectious as dsRNA. Proc. Natl. Acad. Sci. USA 112:9100-9105.

Kazmierczak, P., McCabe, P., Turina, M., Jacob-Wilk, D., and Van Alfen, N. K. 2012. The mycovirus CHV1 disrupts secretion of a developmentally regulated protein in Cryphonectria parasitica. J. Virol. 86:6067-6074.

Kwon, S. J., Cho, S. Y., Lee, K. M., Yu, J., Son, M., and Kim, K. H. 2009. Proteomic analysis of fungal host factors differentially expressed by Fusarium graminearum infected with Fusarium graminearum virus-DK21. Virus Res. 144:96-106.

Lee, K. M., Cho, W. K., Yu, J., Son, M., Choi, H., Min, K., Lee, Y. W., and Kim, K. H. 2014. A comparison of transcriptional patterns and mycological phenotypes following infection of Fusarium graminearum by four mycoviruses. PLoS One 9:e100989.

Lee, K. M., Yu, J., Son, M., Lee, Y. W., and Kim, K. H. 2011. Transmission of Fusarium boothii mycovirus via protoplast fusion causes hypovirulence in other phytopathogenic fungi. PLoS One 6:e21629.

Li, P., Zhang, H., Chen, X., Qiu, D., and Guo, L. 2015. Molecular characterization of a novel hypovirus from the plant pathogenic fungus Fusarium graminearum. Virology 481:151-160.

Lin, H., Lan, X., Liao, H., Parsley, T. B., Nuss, D. L., and Chen, B. 2007. Genome sequence, full-length infectious cDNA clone, and mapping of viral double-stranded RNA accumulation determinant of hypovirus CHV1EP721. J. Virol. 81:1813-1820.

Liu, L., Xie, J., Cheng, J., Fu, Y., Li, G., Yi, X., and Jiang, D. 2014. Fungal negative-stranded RNA virus that is related to bornaviruses and nyaviruses. Proc. Natl. Acad. Sci. USA 111:12205-12210.

Liu, S., Xie, J., Cheng, J., Li, B., Chen, T., Fu, Y., Li, G., Wang, M., Jin, H., Wan, H., and Jiang, D. 2016. Fungal DNA virus infects a mycophagous insect and utilizes it as a transmission vector. Proc. Natl. Acad. Sci. USA 113:12803-12808.

Marzano, S. Y. L., Hobbs, H. A., Nelson, B. D., Hartman, G. L., Eastburn, D. M., McCoppin, N. K., and Domier, L. L. 2015. Transfection of Sclerotinia sclerotiorum with in vitro transcripts of a naturally occurring interspecific recombinant of Sclerotinia sclerotiorum hypovirus 2 significantly reduces virulence of the fungus. J. Virol. 89:5060-5071.

Meister, G., and Tuschl, T. 2004. Mechanisms of gene silencing by doublestranded RNA. Nature 431:343-349.

Milgroom, M. G., and Cortesi, P. 2004. Biological control of chestnut blight with hypovirulence: A critical analysis. Annu. Rev. Phytopathol. 42: 311-338.

Mochama, P., Jadhav, P., Neupane, A., and Lee Marzano, S. Y. 2018. Mycoviruses as triggers and targets of RNA silencing in white mold fungus Sclerotinia sclerotiorum. Viruses 10:214.

Moleleki, N., van Heerden, S. W., Wingfield, M. J., Wingfield, B. D., and Preisig, O. 2003. Transfection of Diaporthe perjuncta with Diaporthe RNA virus. Appl. Environ. Microbiol. 69:3952-3956.

Nguyen, Q., Iritani, A., Ohkita, S., Vu, B. V., Yokoya, K., Matsubara, A., Ikeda, K. I., Suzuki, N., and Nakayashiki, H. 2018. A fungal Argonaute interferes with RNA interference. Nucleic Acids Res. 46:2495-2508.

Nuskern, L., Ježić, M., Liber, Z., Mlinarec, J., and Ćurković-Perica, M. 2018. Cryphonectria hypovirus 1-induced epigenetic changes in infected phytopathogenic fungus Cryphonectria parasitica. Microbiol. Ecol. 75:790-798.

Özkan, S., Mohorianu, I., Xu, P., Dalmay, T., and Coutts, R. H. A. 2017. Profile and functional analysis of small RNAs derived from Aspergillus fumigatus infected with double-stranded RNA mycoviruses. BMC Genomics 18:416.

Pearson, M. N., and Bailey, A. M. 2013. Viruses of Botrytis. Adv. Virus Res. 86:249-272.

Pearson, M. N., Beever, R. E., Boine, B., and Arthur, K. 2009. Mycoviruses of filamentous fungi and their relevance to plant pathology. Mol. Plant Pathol. 10:115-128

Pliego, C., López-Herrera, C., Ramos, C., and Cazorla, F. M. 2012. Developing tools to unravel the biological secrets of Rosellinia necatrix, an emergent threat to woody crops. Mol. Plant Pathol. 13:226-239.

Polashock, J. J., Bedker, P. J., and Hillman, B. I. 1997. Movement of a small mitochondrial double-stranded (ds) RNA element of Cryphonectria 
parasitica: Ascospore inheritance and implications for mitochondrial recombination. Mol. Gen. Genet. 256:566-571.

Rigling, D., and Prospero, S. 2018. Cryphonectria parasitica, the causal agent of chestnut blight: Invasion history, population biology and disease control. Mol. Plant Pathol. 19:7-20.

Robin, C., Lanz, S., Soutrenon, A., and Rigling, D. 2010. Dominance of natural over released biological control agents of the chestnut blight fungus Cryphonectria parasitica in south-eastern France is associated with fitnessrelated traits. Biol. Control 53:55-61.

Root, C., Balbalian, C., Bierman, R., Geletka, L. M., Anagnostakis, S., Double, M., MacDonald, W., and Nuss, D. L. 2005. Multi-seasonal field release and spermatization trials of transgenic hypovirulent strains of Cryphonectria parasitica containing cDNA copies of hypovirus CHV1EP713. For. Pathol. 35:277-297.

Salaipeth, L., Chiba, S., Eusebio-Cope, A., Kanematsu, S., and Suzuki, N. 2014. Biological properties and expression strategy of Rosellinia necatrix megabirnavirus 1 analysed in an experimental host, Cryphonectria parasitica. J. Gen. Virol. 95:740-750.

Sasaki, A., Kanematsu, S., Onoue, M., Oyama, Y., and Yoshida, K. 2006. Infection of Rosellinia necatrix with purified viral particles of a member of Partitiviridae (RnPV1-W8). Arch. Virol. 151:697-707.

Sasaki, A., Onoue, M., Kanematsu, S., Suzaki, K., Miyanishi, M., Suzuki, N., Nuss, D. L., and Yoshida, K. 2002. Extending chestnut blight hypovirus host range within diaporthales by biolistic delivery of viral cDNA. Mol. Plant-Microbe Interact. 15:780-789.

Segers, G. C., van Wezel, R., Zhang, X., Hong, Y., and Nuss, D. L. 2006. Hypovirus papain-like protease p29 suppresses RNA silencing in the natural fungal host and in a heterologous plant system. Eukaryot. Cell 5:896-904.

Segers, G. C., Zhang, X., Deng, F., Sun, Q., and Nuss, D. L. 2007. Evidence that RNA silencing functions as an antiviral defense mechanism in fungi. Proc. Natl. Acad. Sci. USA 104:12902-12906.

Shahi, S., Eusebio-Cope, A., Kondo, H., Hillman, B. I., and Suzuki, N. 2019. Investigation of host range of and host defense against a mitochondrially replicating mitovirus. J. Virol. 93:e01503-e01518.

Stauder, C. M., Nuss, D. L., Zhang, D. X., Double, M. L., MacDonald, W. L., Metheny, A. M., and Kasson, M. T. 2019. Enhanced hypovirus transmission by engineered super donor strains of the chestnut blight fungus, Cryphonectria parasitica, into a natural population of strains exhibiting diverse vegetative compatibility genotypes. Virology 528:1-6.

Sun, L., Nuss, D. L., and Suzuki, N. 2006. Synergism between a mycoreovirus and a hypovirus mediated by the papain-like protease p29 of the prototypic hypovirus CHV1-EP713. J. Gen. Virol. 87:3703-3714.

Sun, Q., Choi, G. H., and Nuss, D. L. 2009. A single Argonaute gene is required for induction of RNA silencing antiviral defense and promotes viral RNA recombination. Proc. Natl. Acad. Sci. USA 106:17927-17932.

Suzuki, N., Maruyama, K., Moriyama, M., and Nuss, D. L. 2003. Hypovirus papain-like protease p29 functions in trans to enhance viral double-stranded RNA accumulation and vertical transmission. J. Virol. 77:11697-11707.

Tomari, Y., and Zamore, P. D. 2005. Perspective: Machines for RNAi. Genes Dev. 19:517-529.

Vainio, E. J., and Hantula, J. 2016. Taxonomy, biogeography and importance of Heterobasidion viruses. Virus Res. 219:2-10.

Vainio, E. J., Jurvansuu, J., Hyder, R., Kashif, M., Piri, T., Tuomivirta, T., Poimala, A., Xu, P., Mäkelä, S., Nitisa, D., and Hantula, J. 2018. Heterobasidion partitivirus 13 mediates severe growth debilitation and major alterations in the gene expression of a fungal forest pathogen. J. Virol. 92: e01744-17.

Vainio, E. J., Piri, T., and Hantula, J. 2013. Virus community dynamics in the conifer pathogenic fungus Heterobasidion parviporum following an artificial introduction of a partitivirus. Microbiol. Ecol. 65:28-38.

van Heerden, S. W., Geletka, L. M., Preisig, O., Nuss, D. L., Wingfield, B. D., and Wingfield, M. J. 2001. Characterization of South African Cryphonectria cubensis isolates infected with a $C$. parasitica hypovirus. Phytopathology 91: 628-632.

Velasco, L., Arjona-Girona, I., Ariza-Fernández, M. T., Cretazzo, E., and López-Herrera, C. 2018. A novel hypovirus species from Xylariaceae fungi infecting avocado. Front. Microbiol. 9:778

Wang, J., Liming, L., He, X., Lu, L., Li, X., and Chen, B. 2016b. Comparative secretome analysis reveals perturbation of host secretion pathways by a hypovirus. Sci. Rep. 6:34308.

Wang, L., Luo, H., Hu, W., Yang, Y., Hong, N., Wang, G., Wang, A., and Wang, L. 2018. De novo transcriptomic assembly and mRNA expression patterns of Botryosphaeria dothidea infection with mycoviruses chrysovirus 1 (BdCV1) and partitivirus 1 (BdPV1). Virol. J. 15:126.

Wang, S., Kondo, H., Liu, L., Guo, L., and Qiu, D. 2013. A novel virus in the family Hypoviridae from the plant pathogenic fungus Fusarium graminearum. Virus Res. 174:69-77.

Wang, S., Li, P., Zhang, J., Qiu, D., and Guo, L. 2016a. Generation of a high resolution map of sRNAs from Fusarium graminearum and analysis of responses to viral infection. Sci. Rep. 6:26151.

Wu, S., Cheng, J., Fu, Y., Chen, T., Jiang, D., Ghabrial, S. A., and Xie, J. 2017. Virus-mediated suppression of host non-self recognition facilitates horizontal transmission of heterologous viruses. PLoS Pathog. 13:e1006234.

Xiao, X., Cheng, J., Tang, J., Fu, Y., Jiang, D., Baker, T. S., Ghabrial, S. A., and Xie, J. 2014. A novel partitivirus that confers hypovirulence on plant pathogenic fungi. J. Virol. 88:10120-10133.

Xie, J., and Jiang, D. 2014. New insights into mycoviruses and exploration for the biological control of crop fungal diseases. Annu. Rev. Phytopathol. 52: 45-68.

Xie, J., Xiao, X., Fu, Y., Liu, H., Cheng, J., Ghabrial, S. A., Li, G., and Jiang, D. 2011. A novel mycovirus closely related to hypoviruses that infect the plant pathogenic fungus Sclerotinia sclerotiorum. Virology 418:49-56.

Yaegashi, H., Kanematsu, S., and Ito, T. 2012. Molecular characterization of a new hypovirus infecting a phytopathogenic fungus, Valsa ceratosperma. Virus Res. 165:143-150.

Yaegashi, H., Nakamura, H., Sawahata, T., Sasaki, A., Iwanami, Y., Ito, T., and Kanematsu, S. 2013a. Appearance of mycovirus-like double-stranded RNAs in the white root rot fungus, Rosellinia necatrix, in an apple orchard. FEMS Microbiol. Ecol. 83:49-62.

Yaegashi, H., Yoshikawa, H., Ito, T., and Kanematsu, S. 2013b. A mycoreovirus suppresses RNA silencing in the white root rot fungus, Rosellinia necatrix. Virology 444:409-416.

Yang, D., Wu, M., Zhang, J., Chen, W., Li, G., and Yang, L. 2018. Sclerotinia minor Endornavirus 1, a novel pathogenicity debilitation-associated mycovirus with a wide spectrum of horizontal transmissibility. Viruses 10:589.

Yu, J., Lee, K. M., Cho, W. K., Park, J. Y., and Kim, K. H. 2018. Differential contribution of RNA interference components in response to distinct Fusarium graminearum virus infections. J. Virol. 92:e01756-e17.

Yu, X., Li, B., Fu, Y., Jiang, D., Ghabrial, S. A., Li, G., Peng, Y., Xie, J., Cheng, J., Huang, J., and Yi, X. 2010. A geminivirus-related DNA mycovirus that confers hypovirulence to a plant pathogenic fungus. Proc. Natl. Acad. Sci. USA 107:8387-8392.

Yu, X., Li, B., Fu, Y., Xie, J., Cheng, J., Ghabrial, S. A., Li, G., Yi, X., and Jiang, D. 2013. Extracellular transmission of a DNA mycovirus and its use as a natural fungicide. Proc. Natl. Acad. Sci. USA 110:1452-1457.

Zhang, D. X., and Nuss, D. L. 2016. Engineering super mycovirus donor strains of chestnut blight fungus by systematic disruption of multilocus vic genes. Proc. Natl. Acad. Sci. USA 113:2062-2067.

Zhang, D. X., Spiering, M. J., Dawe, A. L., and Nuss, D. L. 2014. Vegetative incompatibility loci with dedicated roles in allorecognition restrict mycovirus transmission in chestnut blight fungus. Genetics 197:701-714.

Zhang, X., Shi, D., and Nuss, D. L. 2012. Variation in hypovirus interactions with the fungal-host RNA silencing antiviral-defense response. J. Virol. 86: 12933-12939. 